\title{
ZERO-DISPERSION STOCHASTIC RESONANCE
}

\author{
N G Stocks, N D Stein, S M Soskin ${ }^{1}$ and P V E McClintock \\ School of Physics and Materials, \\ Lancaster University, \\ Lancaster, LA1 4YB.
}

\begin{abstract}
A new form of stochastic resonance (SR), recently discovered in underdamped nonlinear oscillators for which the dependence of eigenfrequency upon energy has an extremum, is investigated. Its characteristic features are identified and discussed on the basis of linear response theory and the fluctuation dissipation theorem. In common with conventional SR (in bistable systems), sharp increases in the response to a weak periodic force, and in the signal/noise ratio, occur with increasing intensity of external noise (temperature) within a certain range. Unlike conventional SR, however, the dependence of the response on frequency is strongly resonant.
\end{abstract}

ICP Numbers: 0540, 0250, 0690

${ }^{1}$ Permanent address: Institute of Semiconductors, Ukrainian Academy of Sciences, Kiev, Ukraine. 
The physical mechanism underlying stochastic resonance (SR) [1-3], in which a weak periodic signal can be optimally amplified by the introduction of external noise of appropriate intensity, is usually assumed to involve fluctuational transitions between the coexisting attractors of systems with double-well (or multi-well) static potentials. We shall refer to phenomena arising through this mechanism as conventional SR. Numerous examples have been found [4] in physics, engineering and biology, as well as in geophysics [2, 3]. The theory of conventional SR has been developed both in terms of Fokker-Planck approximations [5-9] and also through the application of linear response theory (LRT) and the fluctuation dissipation theorem $[10,11]$.

Recently, it has become apparent that SR is actually a much more general phenomenon than had previously been supposed. In particular, it has been shown [12] that significant stochastic enhancements of weak periodic signals can also occur, under appropriate conditions, in underdamped monostable systems. The most pronounced enhancements occurred for a system having an extremum in the dependence of its eigenfrequency upon energy $\omega(E)$, as sketched in Fig 1 . They were observed when the frequency $\Omega$ of the periodic force applied to the system (the signal) was close to the extremal frequency $\omega_{m}=\omega\left(E_{m}\right)$ at which the dispersion $[d \omega(E) / d E]_{E=E_{m}}$ was equal to zero, and the phenomenon was consequently referred to as zero-dispersion stochastic resonance (ZDSR). In this Letter we identify and discuss the characteristic features of ZDSR, the ways in which it differs from conventional SR and, in particular, the extent to which noise-induced enhancements of the signal/noise ratio may be anticipated. We first consider the phenomenon in relation to a general one-dimensional underdamped oscillator with coordinate $q(t)$,

$$
\begin{gathered}
\ddot{q}+2 \Gamma \dot{q}+\frac{d U(q)}{d q}=f(t)+A \cos \Omega t \\
\Gamma \ll 1, \quad\langle f(t)\rangle=0 \\
\left\langle f(t) f\left(t^{\prime}\right)\right\rangle=4 \Gamma T \delta\left(t-t^{\prime}\right)
\end{gathered}
$$

where $f(t)$ is white Gaussian noise of zero mean and intensity $T, \Gamma$ is the damping constant which is assumed small, and $A \cos \Omega t$ is the weak periodic signal whose enhancement will occur under SR conditions. The only restriction placed on the potential $U(q)$ at this stage is that it should be such that $\omega(E)$ will possess at least one extremum, as discussed above. After discussing ZDSR in quite general terms, applicable to any oscillator of this type, we will consider in detail a particular archetypal example of such a system, the tilted single-well Duffing oscillator.

The physical origins of ZDSR can be understood qualitatively in terms of two distinct but linked considerations. First, it is possible for the response to the periodic force to become substantial, even at a frequency $\Omega \simeq \omega_{m}$ different from the oscillator's natural $(E \rightarrow 0)$ frequency $\omega_{0}$, if the system is "tuned" by the introduction of external noise of appropriate intensity $T$ (or equivalently by raising the temperature) such that the probability $\propto e^{-E m / T}$ of its reaching energy $E_{m}$ becomes significant. The response may therefore be expected to increase rapidly with increasing $T$. Secondly, and of central importance for this Letter, the lack of dispersion near $\omega_{m}$ means that fluctuations in the energy of the system will exert only a minimal disruptive influence on the resonance (because the resonant frequency is then virtually energy-independent). 


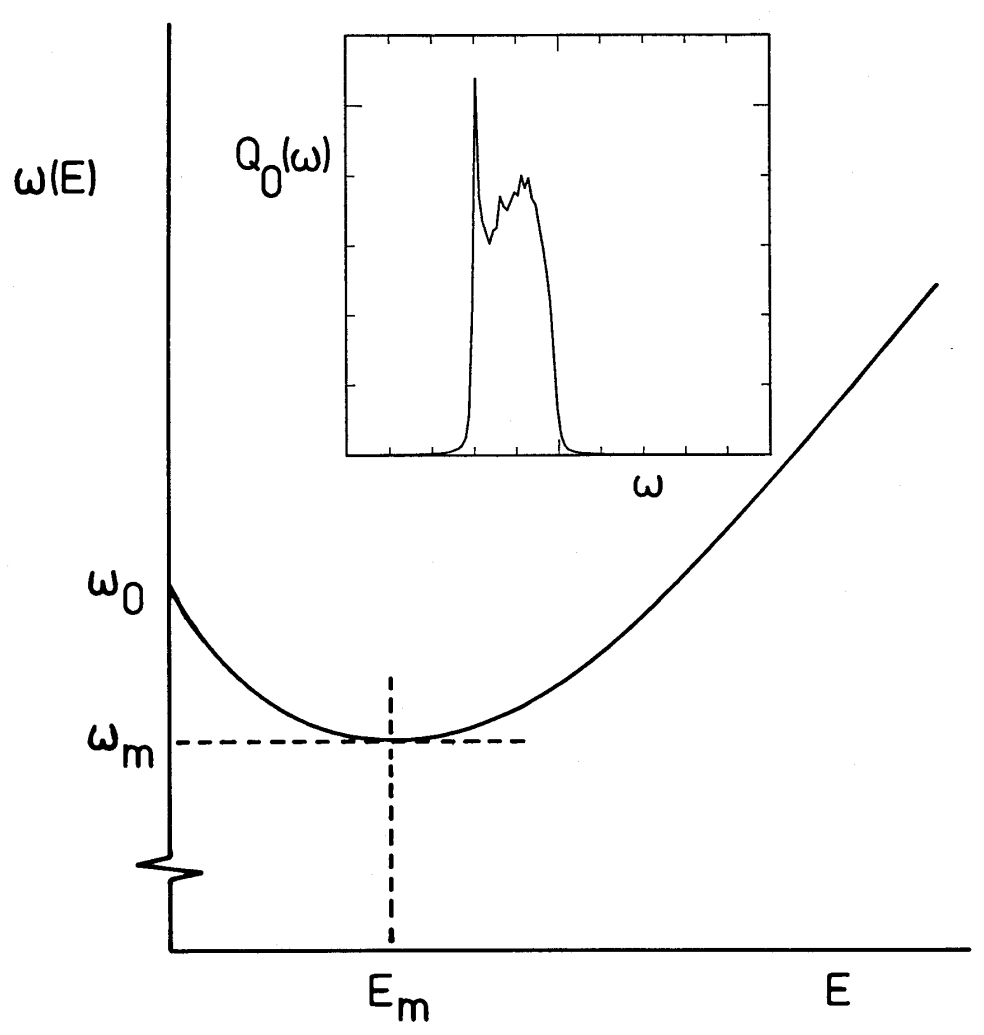

Figure 1: Sketch to illustrate the variation of eigenfrequency $\omega(E)$ with energy $E$ typical of the class of oscillators considered. The eigenfrequency has a minimum value $\omega_{m} \equiv \omega\left(E_{m}\right)$ at energy $E_{m}$. The inset shows a zero-dispersion spectral peak (the sharp spike) appearing in the fluctuation spectrum of the system (1), (11) with $B=2,2 \Gamma=1.7 \times 10^{-3}, T=$ 0.485 measured in the absence of the periodic force $(A=0)$ using an electronic analogue simulator [19].

Provided that the periodic force is weak, a quantitative description of SR in quasithermal equilibrium systems can be developed on the basis of LRT and the fluctuation dissipation theorem [10-12]. We suppose that the ensemble-averaged response of the system to a weak periodic force $A \cos \Omega t$ can be characterized by a susceptibility $\chi(\Omega)$, so that

$$
\langle q(t)\rangle=A \operatorname{Re}\left[\chi(\Omega) e^{-i \Omega t}\right]
$$

where $\chi(\Omega)$ can [13] in turn be expressed in terms of the fluctuation dissipation theorem [14],

$$
\begin{gathered}
\operatorname{Re} \chi(\Omega)=\frac{2}{T} \mathrm{P} \int_{0}^{\infty} d \omega_{1}\left[\omega_{1}^{2} /\left(\omega_{1}^{2}-\Omega^{2}\right)\right] Q_{0}\left(\omega_{1}\right) \\
\operatorname{Im} \chi(\Omega)=(\pi \Omega / T) Q_{0}(\Omega)
\end{gathered}
$$

Here, P implies the Cauchy principal part and

$$
Q_{0}(\omega) \equiv \frac{1}{2 \pi} \int_{-\infty}^{\infty} d t e^{-i \omega t}\langle q(t) q(0)\rangle
$$


is the spectral density of the fluctuations (SDF) of the system in the absence of the periodic force. It has been suggested $[12,15]$ that SR-like phenomena are to be anticipated in any physical system where $Q_{0}(\omega)$ possesses one or more sharp peaks that increase rapidly with increasing $T$. Accordingly, we suppose that $Q_{0}(\omega)$ has a high, narrow, maximum at some frequency $\omega_{m}$. (If the potential resembles that sketched in Fig 1, $\omega_{m}$ will correspond, as above, to the extremal frequency at $E=E_{m}$.) Thus we can write

$$
Q_{0}(\omega)=Q_{\text {peak }}(\omega)+Q_{\text {far }}(\omega)
$$

where $Q_{\text {peak }}(\omega)$ has a maximum of magnitude $Q_{m}$ at $\omega_{m}$ whose width $\Delta \omega \ll \omega_{m}$. We suppose $Q_{\text {far }}(\omega)$ to be negligible at $\omega_{m}$, but that it may still be substantial at frequencies situated far from $\omega_{m}$ in comparison to $\Delta \omega$. If the signal frequency $\Omega \simeq \omega_{m}$, the contributions to $\operatorname{Re}\left[\chi(\Omega)\right.$ ], i.e. to the integral in (3), from frequencies close to $\omega_{m}$ (i.e. for $\left.\left|\omega_{1}-\omega_{m}\right|<\sim \Delta \omega\right)$ and those far from it are respectively

$$
\begin{gathered}
\left.F_{\text {close }} \sim \frac{1}{T} \omega_{m} \frac{d Q}{d \omega}\right|_{\Omega \simeq \omega_{m}} \Delta \omega \sim \frac{\omega_{m} Q_{m}}{T} \\
F_{\text {far }} \sim \frac{1}{T} \frac{\omega_{f}^{2}}{\left|\omega_{f}^{2}-\omega_{m}^{2}\right|} S_{f}
\end{gathered}
$$

where $S_{f}=\int d \omega Q_{f a r}(\omega)$ is the area under the far part of the $\operatorname{SDF} Q_{f a r}(\omega)$, and $\omega_{f}$ lies within the range of the maximum of $Q_{f a r}(\omega)$. If $Q_{f a r}(\omega)$ has several well-defined maxima, $F_{f a r}$ should be a sum of analogous terms for each maximum.

It can be seen from $(7),(8)$ that the contribution to the real part of the susceptibility from frequencies close to $\omega_{m}$ does not depend on the width $\Delta \omega$ of the peak $Q_{\text {peak }}(\omega)$ and that, if the maximum $Q_{m}$ of this peak is high enough, then this contribution will dominate. Correspondingly, given (4) that $\operatorname{Im}\left[\chi\left(\Omega \simeq \omega_{m}\right)\right] \sim Q_{m} \omega_{m} / T$, the absolute magnitude of the response at $\Omega \simeq \omega_{m}$ is given by

$$
|\chi(\Omega)|=\sqrt{[\operatorname{Re}\{\chi(\Omega)\}]^{2}+[\operatorname{Im}\{\chi(\Omega)\}]^{2}} \sim \frac{Q_{m} \omega_{m}}{T}
$$

If $Q_{m}$ increases rapidly with $T$, then the response of the system to a weak periodic force $A \cos \Omega t$ of frequency $\Omega$ close to $\omega_{m}$ will also increase rapidly with $T$. Within the linear response regime, where the signal/noise ratio $[10,11]$ is given by

$$
R=\frac{1}{4} A^{2}|\chi(\Omega)|^{2} / Q_{0}(\Omega)
$$

it is clearly possible for $R$ to rise with increasing $T$ provided only that the increase in $|\chi(\Omega)|^{2}$ with $T$ is sufficiently rapid (i.e. faster than that of $Q_{0}(\Omega)$ ). In the present case, where we assume that the height $Q_{m}$ of the narrow peak in the SDF at $\omega_{m}$ increases very rapidly with $T,(9)$ and (10) clearly demonstrate that stochastic resonance in $R$ is to be expected.

Thus, in the LRT picture, the occurrence of ZDSR stems from the extremely narrow zero dispersion peaks (ZDPs) [16] that arise in the spectral density of the fluctuations of the noise-driven underdamped oscillator (in the absence of the periodic force) whenever the form of $\omega(E)$ has an extremum. For the sake of definiteness, we now consider a particular system of this type, namely the tilted single-well Duffing oscillator, for which the potential function is 


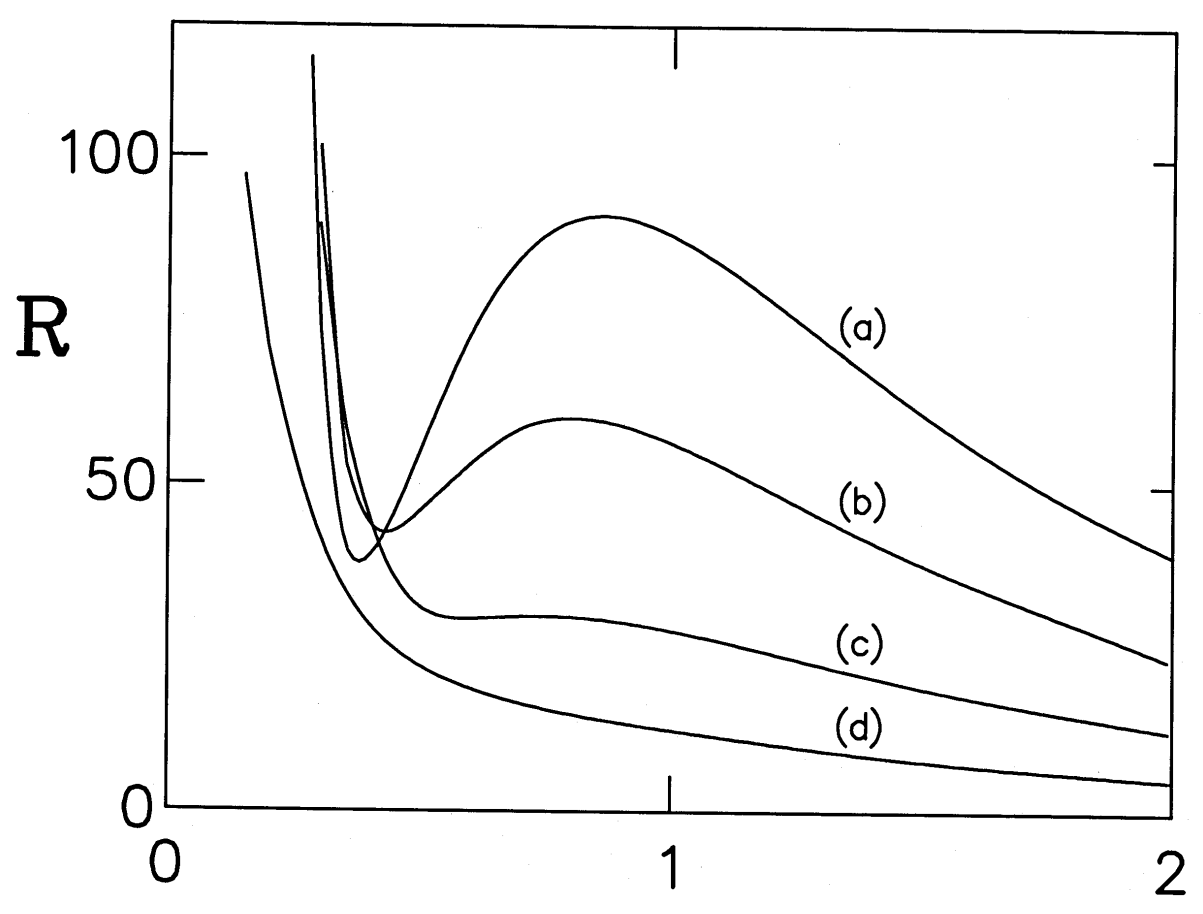

$\mathrm{T}$

Figure 2: The signal/noise ratio $R$ for the system (1) (11), calculated from (3)-(5) and (10) for different values of $\Gamma$ where $Q_{0}(\omega)$ in the absence of the periodic force $(A=0)$ was obtained on the basis of the method given in [17]. The value of $\Omega$ has been chosen in each case to correspond to the maximum of the ZDP. The curves plot $R$ as a function of noise intensity $T$ for: (a) $2 \Gamma=10^{-4}, \Omega=1.794$; (b) $2 \Gamma=10^{-3}, \Omega=1.798$; (c) $2 \Gamma=$ $10^{-2}, \Omega=1.816$; (d) $2 \Gamma=10^{-1}, \Omega=1.846$.

$$
U(q)=\frac{1}{2} q^{2}+\frac{1}{4} q^{4}+B q
$$

In the absence of damping, and with no noise or periodic force, the dependence of the frequency $\omega(E)$ of conservative vibrations on the energy $E=\frac{1}{2} \dot{q}^{2}+U(q)$ possesses a minimum [17], of the form sketched in Fig 1, provided that the modulus of the tilt parameter $|B|>8 /(7)^{\frac{3}{2}} \simeq 0.43$. Correspondingly, a ZDP may be expected to arise at the extremal frequency $\omega_{m}$ in the fluctuation spectrum of the underdamped oscillator (1), (11) with $A=0$, provided that the noise intensity exceeds some critical value, $T_{c} \ll E_{m}$ [18]. For small enough $\Gamma$ the shape of the ZDP is universal, its width $\Delta \omega \propto \Gamma^{\frac{1}{2}}$ and its magnitude $[16,18]$ is

$$
Q^{Z D P}(\Omega)=C_{s} S\left(\frac{\Omega-\omega_{m}}{\Delta \omega \operatorname{sgn}\left[d^{2} \omega\left(E_{m}\right) / d E^{2}\right]}\right)
$$

where $S$ is a universal function and the scaling factor $C_{s} \propto \Gamma^{-\frac{1}{4}} \exp \left(-E_{m} / T\right)$. The ZDP for (1), (11) has recently been observed [19] in analogue electronic experiments (see Fig 1 inset) and found to rise extremely rapidly (exponentially) with increasing $T$, in excellent agreement with the theoretical predictions [16, 18]. 


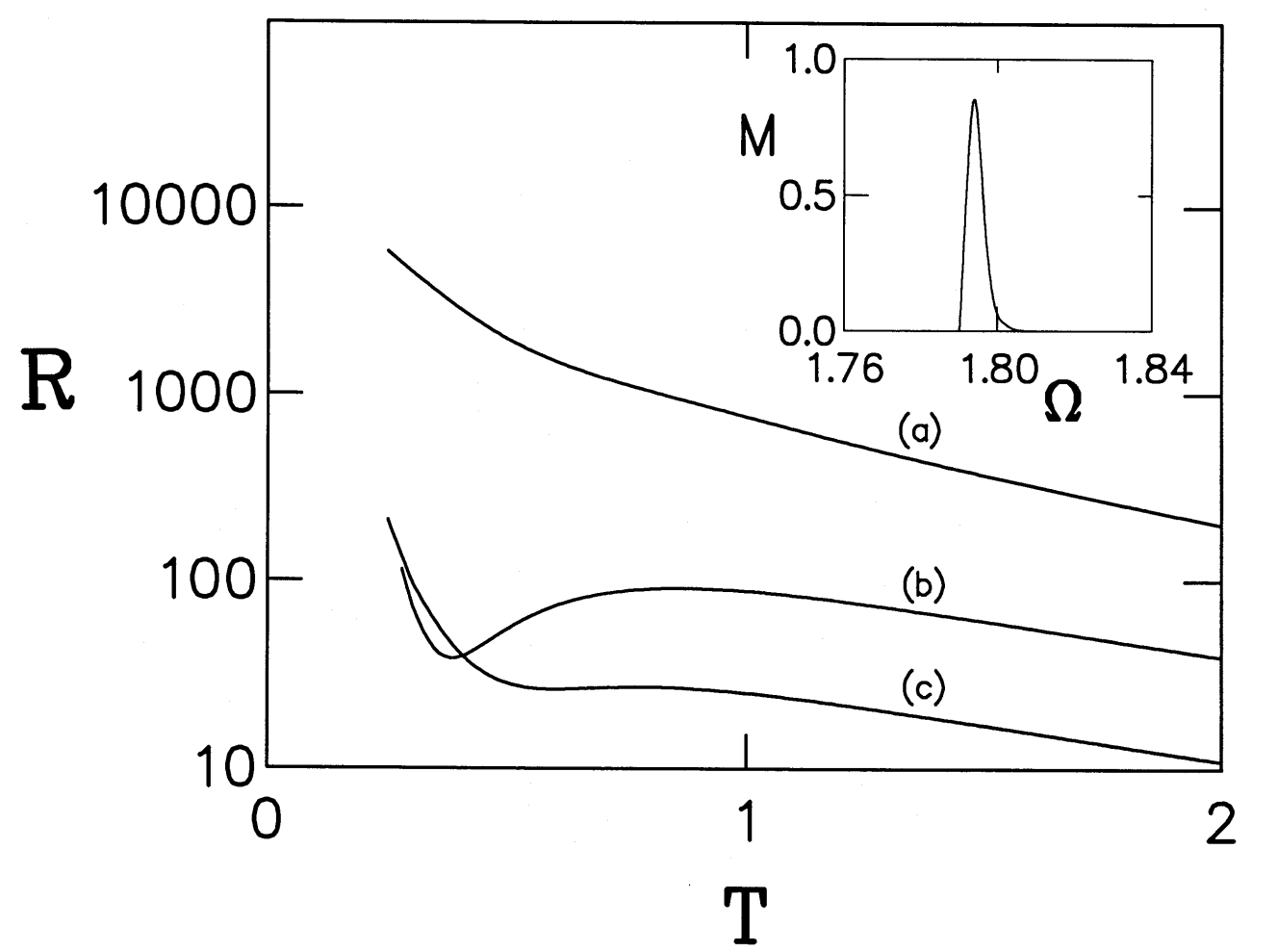

Figure 3: Demonstration of the sensitivity of ZDSR to small changes in the frequency $\Omega$ of the periodic force. The calculated signal/noise ratio $R$ is plotted as a function of noise intensity $T$ for the system (1), (11) with $2 \Gamma=10^{-4}$ and: (a) $\Omega=1.784$; (b) 1.794 ; (c) 1.804. The inset plots the SR magnitude parameter $M$ directly as a function of $\Omega$ for $2 \Gamma$ $=10^{-4}$.

It is evident, therefore, that the system (1), (11) with $|B|>0.43$ meets all the conditions required for the occurrence of ZDSR: in particular, an increase of $R$ with increasing $T$, as well as of $|\chi(\Omega)|$, is to be anticipated. In order to provide a quantitative description on the basis of (3)-(5) and (10), all that we need is the fluctuation spectrum $Q_{0}(\omega)$ of the system in the absence of the periodic force $(A=0)$, which can readily be found numerically using the method given in [17].

Some results of such calculations are shown in Figs 2-4. Figs 2 plots the signal/noise ratio $R$ as a function of noise intensity $T$ for different values of the damping constant $\Gamma$. It is immediately evident that, for large $\Gamma$ as in curve (d), $R$ decreases monotonically with increasing $T$. For smaller $\Gamma$ where the ZDP is more pronounced through $C_{s}$ in (12), on the other hand, there is a range of $T$ for which $R$ increases markedly with increasing $T$, i.e. a strong manifestation of the ZDSR phenomenon is seen for $R$, not just for the response as observed [12] previously. The general shape of $R(T)$, with a rapid rise followed by a slower decrease as $T$ is increased, is similar to that seen in conventional $\mathrm{SR}[1,4,5]$. It is clear that the magnitude of the ZDSR increase of $R$ rises as $\Gamma$ becomes smaller $\left(\propto \Gamma^{-\frac{1}{4}}\right)$, just as expected on the basis of the simple arguments presented above. It may conveniently be characterized in terms of the ratio of the local maximum and local minimum values of $R(T)$,

$$
M=\ln \left(R_{\max } / R_{\text {min }}\right)
$$




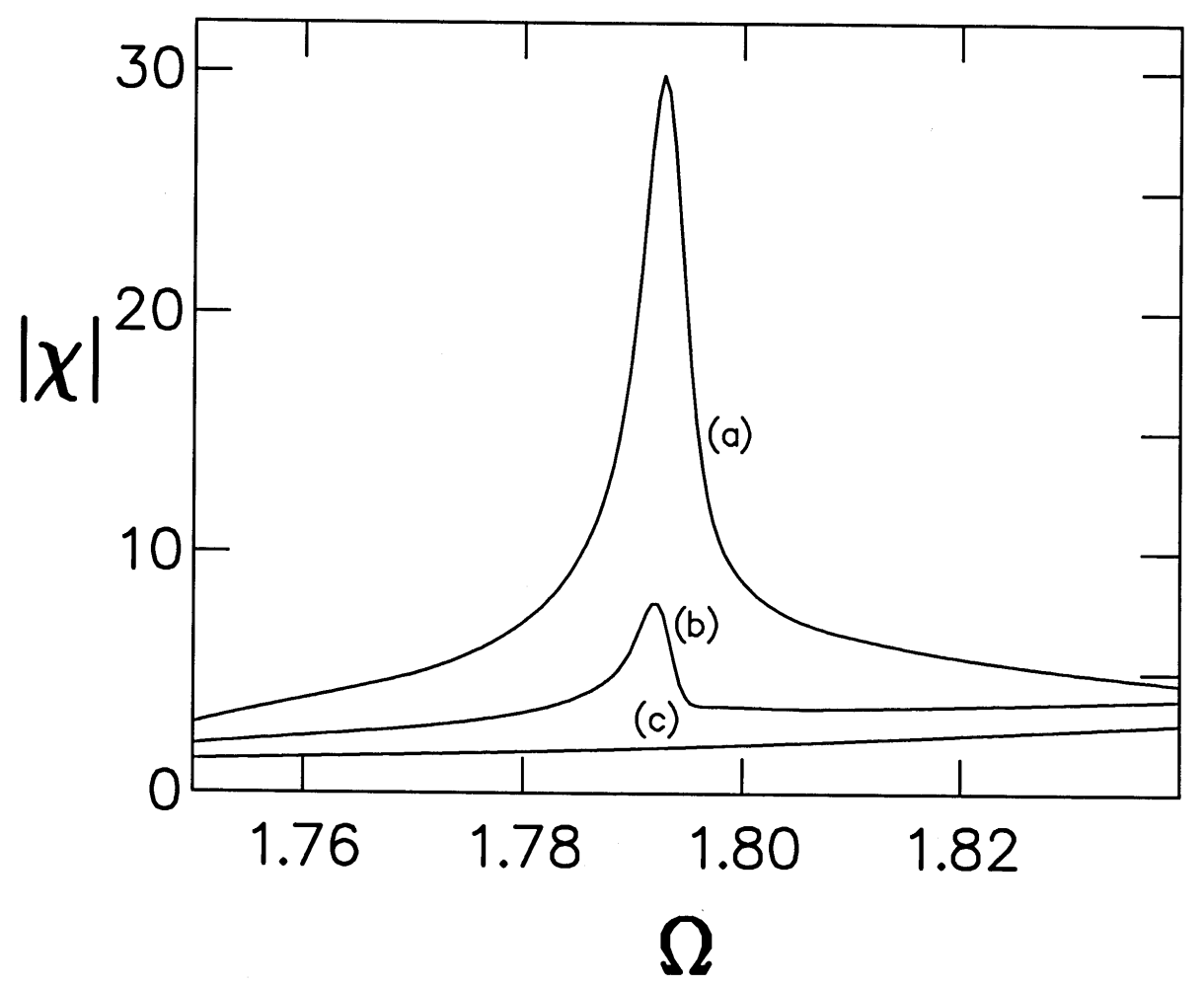

Figure 4: Illustration of the strongly resonant behaviour characteristic of ZDSR. The calculated response $|\chi|$ for the system (1), (11) is plotted directly as a function of the frequency $\Omega$ of the periodic force with $2 \Gamma=10^{-4}$ for three different noise intensities: (a) $T=0.8$; (b) $T=0.4$; (c) $T=0.2$. Note the highly expanded abscissa scale.

where we set $M=0$ when, as e.g. in curve (d), $R(T)$ is monotonic.

It is interesting to compare ZDSR with conventional SR. One of the most marked differences is that ZDSR is a strongly resonant phenomenon. Unless the frequency $\Omega$ of the periodic force is very close to $\omega_{m}, M=0$ i.e. ZDSR does not manifest itself for $R$. Fig 3 shows the effect of small changes in $\Omega$ for $2 \Gamma=10^{-4}$ and it is evident that a change of $\pm 0.6 \%$ in $\Omega$ is enough to eliminate the phenomenon completely; the inset shows how the parameter $M$ varies with $\Omega$. The modulus of the susceptibility $|\chi|$, which determines the response amplitude through (2), is plotted against $\Omega$ (with a highly expanded abscissa scale) for three values of $T$ in Fig 4, demonstrating the resonant character of ZDSR explicitly. This behaviour is in striking contrast to that seen in conventional SR, where the response decreases slowly and monotonically with increasing $\Omega$ [5]. For the particular system (1), (11) and values of $\Gamma$ considered in the present work, the maximum value of the signal/noise enhancement parameter, $M=0.86$ for $2 \Gamma=10^{-4}$, was considerably less than the best results $(M \simeq 2.8)$ achieved in conventional SR [4]; but there is, of course, no limit in principle to the magnitude of $M$ attainable in ZDSR by making $\Gamma$ smaller.

In conclusion we would emphasize that the phenomenon of ZDSR, whose characteristic features are discussed above, is in no way confined to the particular model (1), (11) considered. Rather, ZDSR is a quite general phenomenon to be anticipated in all underdamped oscillators for which the dependence of eigenfrequency on energy possesses one or more extrema $[16,18]$ including, for example, superconducting quantum interference devices (SQUIDs) [20]. 
We freely and warmly acknowledge our substantial indebtedness to Mark Dykman whose highly original perception of SR provided us with the tools needed to develop this work. The research was supported by the Science and Engineering Research Council, by the Royal Society of London, and by the European Community.

\section{References}

1. Benzi R, Sutera A and Vulpiani A, 1981, J. Phys A 14, L453.

2. Nicolis C, 1982, Tellus, 34, 1.

3. Benzi R, Parisi G, Sutera A and Vulpiani A, 1982, Tellus 34, 10.

4. Moss F, 1992, "Stochastic resonance: from the ice ages to the monkey's ear", in Some Problems of Statistical Physics, ed Weiss G H, SIAM, Philadelphia.

5. McNamara B and Wiesenfeld K, 1989, Phys. Rev A 39, 4854.

6. Fox R F, 1989, Phys Rev A 39, 4148.

7. Jung $\mathrm{P}$ and Hanggi P, 1989, Europhys. Lett. 8, 505.

8. Presilla C, Marchesoni F and Gammaitoni L, 1989, Phys Rev A 40, 2105.

9. Gang Hu, Nicolis G and Nicolis C, 1990, Phys Rev A 42, 2030.

10. Dykman M I, McClintock P V E, Mannella R and Stocks N G, 1990, Sov. Phys. JETP Lett 52, 141.

11. Dykman M I, McClintock P V E, Mannella R and Stocks N G, 1992, Phys Rev Lett 68, 2985.

12. Stocks N G, Stein N D and McClintock P V E, "Stochastic resonance in monostable systems", to be published.

13. Risken H, 1989, The Fokker-Planck Equation, 2nd edn, Springer, Berlin.

14. Landau L D and Lifshitz E M, 1980, Statistical Physics, 3rd edn, Pergamon, New York.

15. Dykman M I, Luchinsky D G, Mannella R, McClintock P V E, Stein N D and Stocks N G, "Non-conventional stochastic resonance", J Stat Phys, in press.

16. Soskin S M, 1989, Physica A 155401.

17. Dykman M I, Mannella R, McClintock P V E, Soskin S M and Stocks N G, 1990, Phys Rev A 42, 7041.

18. Soskin S M, 1992, Physica A 180, 386.

19. Stocks N G, McClintock P V E and Soskin S M, "Observation of zero-dispersion peaks in the fluctuation spectrum of an underdamped single-well oscillator", to be published.

20. Barone R and Paterno G, 1982 Physics and Applications of the Josephson Effect, Wiley, New York. 